\title{
Advances in pharmacotherapy of cataracts
}

\author{
Jingjie Xu ${ }^{1}$, Qiuli Fu' ${ }^{1}$, Xiangjun Chen ${ }^{1,2}$, Ke Yao ${ }^{1}$ \\ ${ }^{1}$ Eye Center of the Second Affiliated Hospital, Medical College of Zhejiang University, Hangzhou, China; ${ }^{2}$ Institute of Translational Medicine, \\ Zhejiang University School of Medicine, Hangzhou, China \\ Contributions: (I) Conception and design: All authors; (II) Administrative support: K Yao, X Chen; (III) Provision of study materials or patients: None; \\ (IV) Collection and assembly of data: All authors; (V) Data analysis and interpretation: All authors; (VI) Manuscript writing: All authors; (VII) Final \\ approval of manuscript: All authors. \\ Correspondence to: Ke Yao. Eye Center of the Second Affiliated Hospital, Zhejiang University School of Medicine, 310009 Hangzhou, China. \\ Email: xlren@zju.edu.cn; Xiangjun Chen. Eye Center of the Second Affiliated Hospital, Institute of Translational Medicine, Zhejiang University \\ School of Medicine, 310009 Hangzhou, China. Email: chenxiangjun@zju.edu.cn.
}

\begin{abstract}
Cataracts, the leading cause of vision impairment worldwide, arise from abnormal aggregation of lens proteins. According to the World Health Organization, cataracts cause more than $40 \%$ of blindness cases. As the population ages, the prevalence of cataracts will increase rapidly. Although cataract surgery is regarded as effective, it still suffers from complications and high cost, and could not meet the increasingly surgery demand. Therefore, pharmacological treatment for cataracts is a cheaper and more readily available option for patients, which is also a hot topic for years. Anti-cataract drug screening was previously mainly based on the specific pathogenic factors: oxidative stress, excess of quinoid substances, and aldose reductase (AR) activation. And several anti-cataract drugs have been applied in the clinic, while the effect is still unsatisfied. Makley and Zhao recently identified two kinds of novel pharmacological substances (25-hydroxycholesterol, lanosterol) that can reverse lens opacity by dissolving the aggregation of crystallin proteins, indicating that protein aggregation is not an endpoint and could be reversed with specific smallmolecule drugs, significantly boosting the development of the cataract pharmacopeia and being regarded as a new dawn for cataract treatment. Our team built a novel optimized platform and had screened several potential therapeutic agents from a collection of lanosterol derivatives. In this review, we would mainly focus on the advancement of cataract pharmacotherapy based on the targets for anti-cataract drugs.
\end{abstract}

Keywords: Cataract; lens transparency; anti-cataract drug; crystallin aggregates; lanosterol derivatives

Submitted Feb 26, 2020. Accepted for publication Jul 10, 2020.

doi: $10.21037 /$ atm-20-1960

View this article at: http://dx.doi.org/10.21037/atm-20-1960

\section{Introduction}

Cataracts, which appear as an opacity of the lens, are the leading cause of visual impairment. According to etiology, cataracts can be divided into two types: (I) congenital and (II) acquired. The former is mainly related to inherited tendencies. The latter is associated with aging, genetics, diseases, drugs, smoking, or UV exposure, which also the common risk factors for cataracts $(1,2)$. The prevalence of cataracts is around $47.8 \%$ among the people who over 50 years of age and cataracts afflict millions of people worldwide (3). Besides, according to the World Health
Organization, cataracts cause more than $40 \%$ of blindness cases $(3,4)$, and cause approximately $90 \%$ of blindness in developed countries (1). Moreover, literature dated that direct medical costs for cataracts were around $\$ 6.8$ billion annually in the US in 2008 (1) and still increasing continusely, which also happened in other countries and brought a significant economic burden. Nowadays, with a growing population and prolonged lifespans, the number of people with cataracts will inevitably increase in the future, which will bring an even greater burden from cataracts.

Surgery is currently the most direct and effective treatment for cataracts. Opaque lenses are removed 
by phacoemulsification and replaced with artificial lenses. Although surgery is efficient, it still suffers from complications, such as postsurgical inflammation, intraocular lens dislocation, and posterior capsular opacity (5). In addition, the high cost and technical requirements limit the availability of cataract surgery, especially in areas where financial resources and medical facilities are limited $(6,7)$.

Pharmacological therapy is therefore an ideal choice for cataracts prevention and treatment, which also has both a lower economic burden and higher availability. Drug screening research for cataracts has attracted a lot of attention, and some recent studies have reported several compounds that could prevent and dissolve protein aggregates in the lens, offering a novel anti-cataract drug screening strategy $(8,9)$. In this article, we will review the development and application of anti-cataract drugs based on their various targets (Table 1).

\section{Drug screening based on the specific pathogenic factors of cataracts}

Oxidative stress, excess of quinoid substances, and aldose reductase (AR) activation are essential in the progression of cataracts. Researchers have screened some potential drugs for cataract prevention based on these three factors, and the following will introduce such potential and classical anticataract drugs.

\section{Anti-cataract drug screening based on the oxidative/ antioxidative system}

Oxidative stress is regarded as having an essential role in the initiation and development of cataracts $(21,22)$. Numerous studies have reported a higher level of membrane lipid peroxidation and reactive oxygen species (ROS) in cataract tissue than in normal tissue $(23,24)$. Hydrogen peroxide $\left(\mathrm{H}_{2} \mathrm{O}_{2}\right)$, a principal oxidative substance in the lens and aqueous humor, is found at high levels in cataract samplesas much as 30 times that in normal samples (25). Such increased ROSs induce apoptosis of lens epithelial cells and the denaturing of crystallin proteins, which promote the solubility loss of crystallin and the formation of aggregations $(26,27)$.

Consequently, antioxidants and ROS scavengers have a potential therapeutic effect for cataracts. Glutathione (GSH), which is enriched in the lens, plays a vital role in inhibiting oxidative stress (28), and numerous studies have suggested that upregulating GSH and GSH synthesis enzymes could prevent cataract formation (10). There is evidence that GSH and L-cystine, as potential drugs, could protect the lens from ROSs and lipid peroxidation by elevating the activity of GSH and GSH-RX in animal testing (11), and lutein, zeaxanthin, vitamin $\mathrm{E} / \mathrm{C}$, and carotenoids have also been used as antioxidants to prevent cataracts (12-14). Numerous experiments have clearly shown the positive effects of these antioxidants against cataracts (29-31), and exogenous supplementation with these reagents has shown a clear positive effect in retarding the development of cataracts (31-33). Some eye drops, such as Vita-Iodurol (France) and Quinax (USA), have been used in the clinic since the 1980s (15), and some clinical trial evidence has indicated that these drugs could prevent the early development of cataracts, but they are ineffective in patients with advanced diabetes (34-36).

\section{Anti-cataract drug screening based on quinoid substances}

The quinones are also critical in the development of cataracts. In living systems, quinones and amino acids are considered separated and do not work together $(37,38)$. A low concentration of quinone could react with proteins and enzymes, which could lead to a deleterious effect. Naphthalene-a reagent that can be oxidized to reactive quinone - can induce cataracts in rabbits at a dose of $1 \mathrm{~g} / \mathrm{KG}$ by reacting with lens protein and promoting aggregation (39). The SH group of lens proteins can be oxidized to SS bonds by quinone and disturb the interaction balance of lens proteins, causing the aggregation of proteins. Catalin (pirenoxine)-a commercial eye drop that was introduced in 1958 to prevent early cataracts—can competitively inhibit the sulfhydryl combination of quinoid substances with lens proteins and maintain the transference of the lens (40). Kociecki's and Hu's experiments have also revealed that Catalin eye drops are effective in protecting against UVC-, selenite-, and calcium-induced lens opacification, especially in the early stages $(40,41)$.

\section{Anti-cataract drug screening based on AR}

AR can catalyze the conversion of glucose to sorbitol by the polyol pathway, initiating the formation of cataractsespecially diabetic cataracts. Typically, the majority of glucose metabolism is through glycolysis and little is through the polyol pathway $(17,42,43)$, while, in hyperglycemia, more than $30 \%$ of glucose is converted to sorbitol by catalysis with AR, leading to the accumulation of sorbitol and fructose. Several experiments have suggested that an increasing level of sorbitol and fructose aggravates the 
Table 1 Summary of drug candidates

\begin{tabular}{|c|c|c|c|c|}
\hline Class & Drug/compound & Action & Effect & Ref \\
\hline \multirow{6}{*}{$\begin{array}{l}\text { Oxidative/antioxidative } \\
\text { system }\end{array}$} & L-cystine & \multirow{6}{*}{$\begin{array}{l}\text { Protect the lens from ROSs and lipid peroxidation } \\
\text { by elevating the activity of GSH and GSH-RX }\end{array}$} & \multirow{6}{*}{$\begin{array}{l}\text { Effective in early cataract, } \\
\text { ineffective in diabetic } \\
\text { cataract }\end{array}$} & (11) \\
\hline & Lutein & & & $(12-14)$ \\
\hline & Zeaxanthin & & & \\
\hline & Carotenoids & & & \\
\hline & Vita-lodurol (France) & & & $(15)$ \\
\hline & Quinax (USA) & & & \\
\hline Anti-aldose reductase & Bendazac lysine & Anti-denaturant effect on proteins & Effective in early cataracts & $(16)$ \\
\hline \multirow{2}{*}{$\begin{array}{l}\text { Dissolving crystallin } \\
\text { aggregates }\end{array}$} & Lanosterol & Solubilize the aggregates of crystallin proteins & $\begin{array}{l}\text { Evidenced in the } \\
\text { cataractous dog }\end{array}$ & (19) \\
\hline & Rosmarinic acid & Remodeling lentic protein aggregates & Evidenced in vitro & $(20)$ \\
\hline
\end{tabular}

lenticular opacity by increasing cellular osmotic stress and oxidative stress, giving rise to cataractogenesis (42,44-46).

However, AR inhibition effectively inhibits epithelialmesenchymal transition but does not inhibit the differentiation of fiber cells, which are also essential in cataractogenesis (47). Thus, AR inhibitors are considered a potential drug for cataract treatment, especially for hyperglycemia patients, and the screening of AR inhibitors has therefore attracted attention. Bendazac lysine, an AR inhibitor, has an anti-denaturant effect on protein and has been used for decades to prevent early cataracts (16), and several researchers have reported a positive effect on lens transparency at a dosage of $500 \mathrm{mg}$ orally three times daily. A double-masked placebocontrolled clinical trial has also shown that Bendazac lysine eyedrops $0.5 \%$, at a dosage of two drops three times daily, can slow the progression of cataracts (16), and a further study has recently shown that diosgenin, a novel AR inhibitor, can markedly decrease the lens epithelial cells' osmotic expansion and delay the progression of rat cataracts (17).

\section{Anti-cataract drug development based on dissolving crystallin aggregates}

Aggregation of misfolded lentic proteins is the common cause of different types of cataract $(48,49)$. The misfolded proteins alter the interaction of lentic proteins and significantly decrease the solubility and stability of crystallin proteins, thus promoting the formation of aggregations and lens opacity. In the past, despite nutrient supplementation and many drugs having been screened and used to manage cataracts, it was only possible to slow the progression of the disease but not to reverse the process of lens opacity. However, Makley et al. and Zhao et al. discovered that 5-cholesten-3b,25-diol and lanosterol, respectively, could effectively reverse clouded lenses to transparency $(8,18,19)$. These findings indicate that protein aggregation is not an endpoint and could be reversed with specific small-molecule drugs, significantly boosting the development of the cataract pharmacopeia and being regarded as a new dawn for cataract treatment (50).

\section{High-throughput drug screening platforms for anti- cataract drugs}

The creation of high-throughput drug screening platforms has played an essential role in drug screening and development, allowing researchers to find the small numbers of useful compounds from among millions. One representative 
screening system is the high-throughput differential scanning fluorimetry platform built by Makley (18), which found the molecule 5-cholesten-3b,25-diol. They used Hsp27-a hot-shock protein that consists of a highly conserved crystallin domain-as the model protein to pilot screen for its superior signal. However, 5-cholesten-3b,25diol was only valid for cataracts caused by $\alpha$-crystallin mutations, so this screening method had certain limitations. A novel optimized platform (Figure 1) has since been created for the systematic screening of potential therapeutic agents; that ex vivo platform examines human lens particles removed from patients during routine cataract surgery that are treated with one of several protein aggregation modulators. This innovative, yet straightforward, experimental approach has enabled us to directly test the impact of screened compounds on protein aggregates present in phacoemulsified human crystalline lens material, and our team has screened a collection of lanosterol derivatives and identified several potential small molecules.

\section{Potential candidates for pharmacological cataract treatment}

5-cholesten-3b,25-diol (25-hydroxycholesterol), a novel pharmacological chaperone, is a small molecule that could stabilize the native state of a protein. Using highthroughput differential scanning fluorimetry, Makley determined that this compound could activate HSP27 (18), suggesting that 5 -cholesten-3b,25-diol could protect the $\alpha B$-crystallin from misfolding and maintain the solubility of other crystallin proteins. However, the mechanism still needs to be explored further.

Lanosterol is present in the non-saponification of lanolin, is the intermediate in cholesterol biosynthesis, and is formed by lanosterol synthase (LSS). In our study, we found two congenital cataract families with a mutation in LSS and determined that lanosterol could solubilize the aggregates of crystallin proteins in vitro and in cells and could even reverse the opacity of the lens in dogs and rabbits with age-related cataracts. Unlike 25-hydroxycholesterol, which can only maintain the native status of $\alpha$-crystallin, lanosterol can restore and dissolve $\alpha$ - and $\beta / \gamma$-crystallin proteins. The potential mechanism revealed by molecular simulation suggests that lanosterol preferentially binds to the hydrophobic dimerization interface in the C-terminus domain of $\gamma \mathrm{D}$-crystallin proteins and protects from aggregation and cataract formation (51). Lanosterol is also thought to enhance the activity of proteasome and to promote the clearance of misfolding or aggregated proteins (52).

Although lanosterol can efficiently dissolve aggregates, the pharmacokinetics are still problematic in several ways. One is that lanosterol exists naturally and involves in the metabolism of lipids into cholesterol, making it difficult to maintain sufficient concentrations in the lens. Another is that its low solubility and activity make it hard to use in the clinic. Our group therefore designed and synthesized 45 derivatives of lanosterol to find a more effective option. Experiments showed that lanosterol derivatives could efficiently reverse multiple protein aggregations using a mutant crystallin cell line or ex vivo screening system. Excitingly, some derivatives, such as C6 and C34, possess both higher solubility and vitality — thus making them more efficient-than lanosterol.

A recent study also suggests that rosmarinic acid can restore clarity of the lens by remodeling lentic protein aggregates (20). Interestingly, rosmarinic acid has also been shown to prevent misfolding- and aggregate-related neurogenerative diseases by directly interfering with early oligomers of amyloid-beta and alpha-synuclein (53-56). This also shows that focusing on dissolving aggregates is a practical way to screen and develop cataract drugs.

Although the above results are exciting, several issues still require attention. One is that the safety of these compounds in humans remains unknown. For instance, lanosterol could convert to cholesterol, which would harm the cardiovascular system (57), meaning that lanosterol therapy might lead to high hypercholesteremia and systemic side effects. Therefore, further research is needed to establish efficacy and safety profiles. A second issue is that the low solubility of lanosterol limits its medicinal properties. Thus, a proper drug delivery strategy should be identified that can increase the solubility and enhance the anti-cataract efficacy of lanosterol. For example, nanotechnology could offer more efficient drug delivery to the eye than nanoparticles, micelles, dendrimers, or liposomes (58).

\section{Conclusions}

Cataracts are the leading cause of visual recession worldwide. Despite the rapid development of cataract surgery technology over the years, surgery remains a challenge due to its cost and the increasing number of patients, especially in developing countries. Pharmacologic therapy may therefore be an efficient method to reduce the disease burden and medical costs and to benefit patients in rural areas (9). Over the last few decades, numerous 


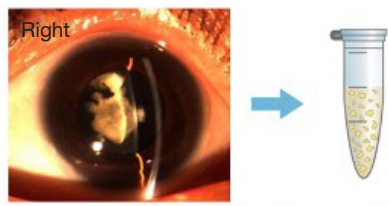

Surgical removal from cataractous lens
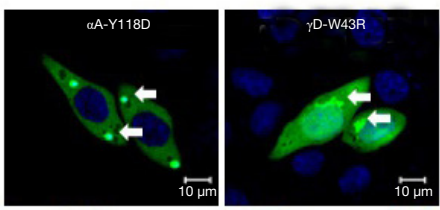

Cell with crystallin mutation

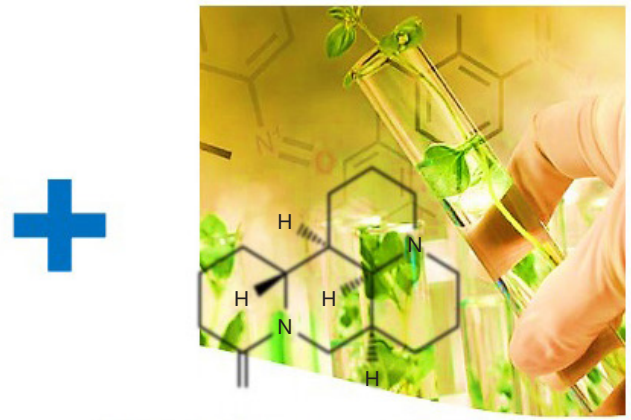

Candidate compounds library

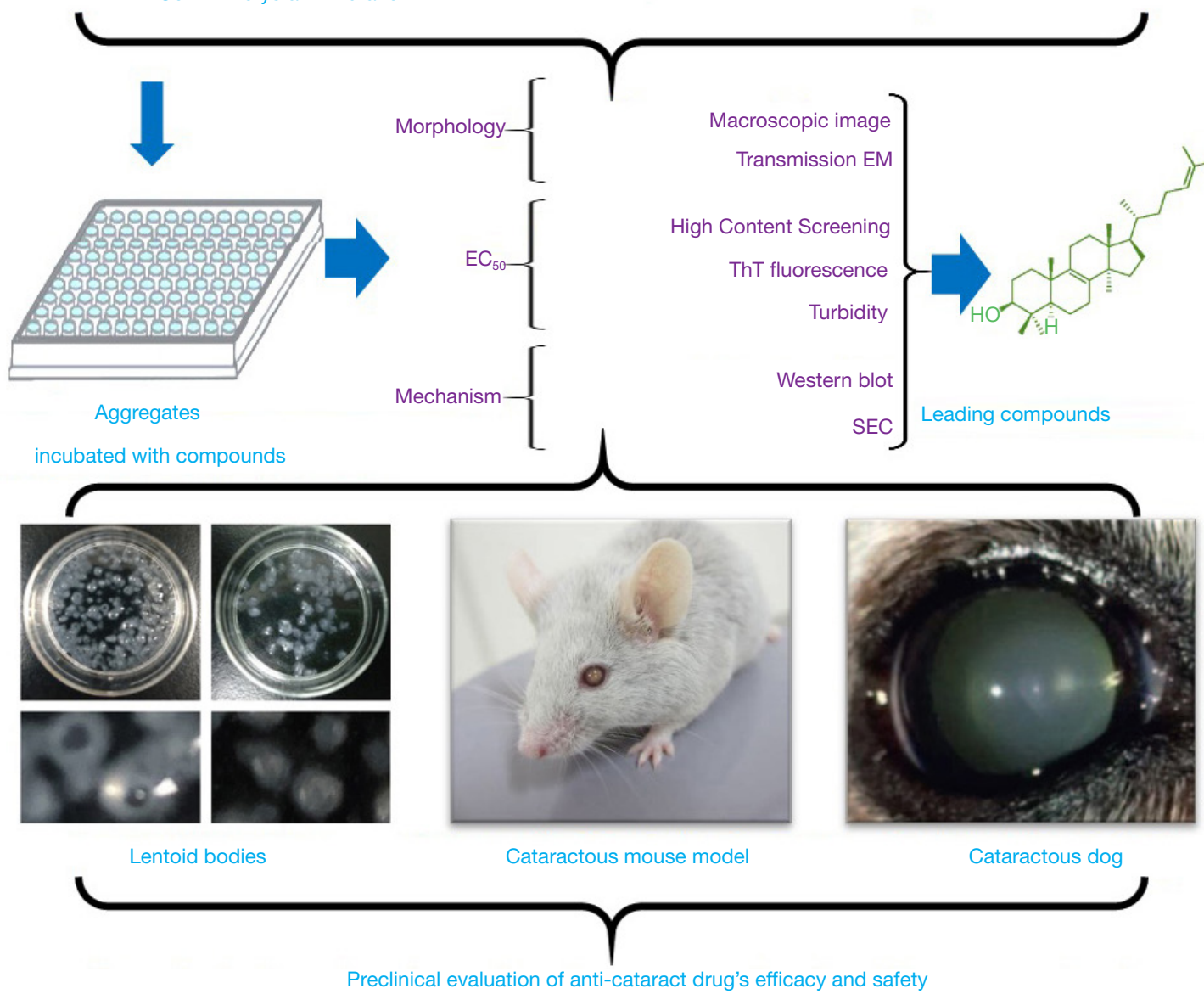

Figure 1 A novel high-throughput drug-screening platform for anti-cataract drugs. The human aggregates samples were surgical removed from cataractous lens and the cellular samples were collected from the cell with crystallin mutation. The aggregates samples were then incubated with chemicals from the candidate compounds library. In order to select a proper drugs, we first evaluated the effects from morphology, EC50 (concentration for 50\% of maximal effect), and the mechanism. The macroscopy and transmission electron microscope (TME) were applied to observe the morphology profiles. High content screening (HCS), ThT fluorescence, and UV (to test the turbidity) were used to calculate the EC50. Western-blotting and size-exclusion chromatography (SEC) were used to explore the mechanism. Finally, the efficacy and safety of selected compounds were estimated in lentoid bodies, cataractous mouse model, and cataractous dog model. 
studies have focused on pharmacological strategies for preventing and retarding the progression of cataracts using antioxidants, AR inhibitors, and sulfhydryl combinations of quinoid substance inhibitors, without ultimately influencing the endpoint (59). Excitingly, 25-hydroxycholesterol, lanosterol, and lanosterol derivatives have now been discovered and may reverse lens opacity, creating a new way to prevent and cure cataracts. However, further work is needed to explore the exact mechanisms involved in the formation of cataracts and the regulation of protein homeostasis in the lens, which will help in finding new therapeutic targets and new candidate compounds for cataracts and thus impact the global morbidity associated with this major public health concern.

\section{Acknowledgments}

The authors thank Professors Yongbin Yan (Tsinghua University) and Yu Rao (Tsinghua University) for helpful suggestions.

Funding: This study was supported by the National Natural Science Foundation of China (No. 31872724, No. 81700815, No. 81870641, and No. 81900837) and Zhejiang Province Key Research and Development Program (No. 2019C03091).

\section{Footnote}

Provenance and Peer Review: This article was commissioned by the Guest Editor (Dr. Andrzej Grzybowski) for the series "Recent developments in cataract surgery" published in Annals of Translational Medicine. The article was sent for external peer review organized by the Guest Editor and the editorial office.

Conflicts of Interest: All authors have completed the ICMJE uniform disclosure form (available at http:// dx.doi.org/10.21037/atm-20-1960). The series "Recent developments in cataract surgery" was commissioned by the editorial office without any funding or sponsorship. The authors report grants from National Natural Science Foundation of China, grants from Zhejiang Province Key Research and Development Program, during the conduct of the study.

Ethical Statement: The authors are accountable for all aspects of the work in ensuring that questions related to the accuracy or integrity of any part of the work are appropriately investigated and resolved.

Open Access Statement: This is an Open Access article distributed in accordance with the Creative Commons Attribution-NonCommercial-NoDerivs 4.0 International License (CC BY-NC-ND 4.0), which permits the noncommercial replication and distribution of the article with the strict proviso that no changes or edits are made and the original work is properly cited (including links to both the formal publication through the relevant DOI and the license). See: https://creativecommons.org/licenses/by-nc-nd/4.0/.

\section{References}

1. Thompson J, Lakhani N. Cataracts. Prim Care 2015;42:409-23.

2. Gupta SK, Selvan VK, Agrawal SS, et al. Advances in pharmacological strategies for the prevention of cataract development. Indian J Ophthalmol 2009;57:175-83.

3. Prokofyeva E, Wegener A, Zrenner E. Cataract prevalence and prevention in Europe: A literature review. Acta Ophthalmol 2013;91:395-405.

4. Lee CM, Afshari NA. The global state of cataract blindness. Curr Opin Ophthalmol 2017;28:98-103.

5. Goodman DF, Stark WJ, Gottsch JD. Complications of cataract extraction with intraocular lens implantation. Ophthalmic Surg 1989;20:132-40.

6. Lian RR, Afshari NA. The quest for homeopathic and nonsurgical cataract treatment. Curr Opin Ophthalmol 2020;31:61-6.

7. Wang W, Yan W, Fotis K, et al. Cataract Surgical Rate and Socioeconomics: A Global Study. Invest Ophthalmol Vis Sci 2016;57:5872-81.

8. Quinlan RA. DRUG DISCOVERY. A new dawn for cataracts. Science 2015;350:636-7.

9. Skinner C, Miraldi Utz V. Pharmacological approaches to restoring lens transparency: Real world applications. Ophthalmic Genet 2017;38:201-5.

10. Whitson JA, Wilmarth PA, Klimek J, et al. Proteomic analysis of the glutathione-deficient LEGSKO mouse lens reveals activation of EMT signaling, loss of lens specific markers, and changes in stress response proteins. Free Radic Biol Med 2017;113:84-96.

11. Babizhayev MA. Generation of reactive oxygen species in the anterior eye segment. Synergistic codrugs of $\mathrm{N}$-acetylcarnosine lubricant eye drops and mitochondriatargeted antioxidant act as a powerful therapeutic platform for the treatment of cataracts and primary open-angle 
glaucoma. BBA Clin 2016;6:49-68.

12. Christen WG, Liu S, Glynn RJ, et al. Dietary carotenoids, vitamins $\mathrm{C}$ and $\mathrm{E}$, and risk of cataract in women: $\mathrm{A}$ prospective study. Arch Ophthalmol 2008;126:102-9.

13. Jiang H, Yin Y, Wu CR, et al. Dietary vitamin and carotenoid intake and risk of age-related cataract. Am J Clin Nutr 2019;109:43-54.

14. Zhang Y, Jiang W, Xie Z, et al. Vitamin E and risk of agerelated cataract: A meta-analysis. Public Health Nutr 2015;18:2804-14.

15. Chasovnikova LV, Formazyuk VE, Sergienko VI, et al. The antioxidative properties of carnosine and other drugs. Biochem Int 1990;20:1097-103.

16. Balfour JA, Clissold SP. Bendazac Lysine: A Review of its Pharmacological Properties and Therapeutic Potential in the Management of Cataracts. Drugs 1990;39:575-96.

17. Ji L, Cheng L, Yang Z. Diosgenin, a Novel Aldose Reductase Inhibitor, Attenuates the Galactosemic Cataract in Rats. J Diabetes Res 2017;2017:7309816.

18. Makley LN, McMenimen KA, DeVree BT, et al. Pharmacological chaperone for $\alpha$-crystallin partially restores transparency in cataract models. Science 2015;350:674-7.

19. Zhao L, Chen XJ, Zhu J, et al. Lanosterol reverses protein aggregation in cataracts. Nature 2015;523:607-11.

20. Chemerovski-Glikman M, Mimouni M, Dagan Y, et al. Rosmarinic acid restores complete transparency of sonicated human cataract ex vivo and delays cataract formation in vivo. Sci Rep 2018;8:9341.

21. Chen H, Zhou J. Effects of Sodium Selenite on Oxidative Damage in the Liver, Kidney and Brain in a Selenite Cataract Rat Model. Biol Trace Elem Res 2020;197:533-43.

22. Lou MF. Redox regulation in the lens. Prog Retin Eye Res 2003;22:657-82.

23. Nita M, Grzybowski A. The Role of the Reactive Oxygen Species and Oxidative Stress in the Pathomechanism of the Age-Related Ocular Diseases and Other Pathologies of the Anterior and Posterior Eye Segments in Adults. Oxid Med Cell Longev 2016;2016:3164734.

24. Babizhayev MA. Mitochondria induce oxidative stress, generation of reactive oxygen species and redox state unbalance of the eye lens leading to human cataract formation: disruption of redox lens organization by phospholipid hydroperoxides as a common basis for cataract disease. Cell Biochem Funct 2011;29:183-206.

25. Spector A. Oxidative stress-induced cataract: mechanism of action. FASEB J 1995;9:1173-82.
26. Mulhern ML, Madson CJ, Danford A, et al. The unfolded protein response in lens epithelial cells from galactosemic rat lenses. Invest Ophthalmol Vis Sci 2006;47:3951-9.

27. Berthoud VM, Beyer EC. Oxidative stress, lens gap junctions, and cataracts. Antioxid Redox Signal 2009;11:339-53.

28. Giblin FJ. Glutathione: a vital lens antioxidant. J Ocul Pharmacol Ther 2000;16:121-35.

29. Thiagarajan R, Manikandan R. Antioxidants and cataract. Free Radic Res 2013;47:337-45.

30. Sedaghat F, Ghanavati M, Nezhad Hajian P, et al. Nutrient patterns and risk of cataract: a case-control study. Int J Ophthalmol 2017;10:586-92.

31. McCusker MM, Durrani K, Payette MJ, et al. An eye on nutrition: The role of vitamins, essential fatty acids, and antioxidants in age-related macular degeneration, dry eye syndrome, and cataract. Clin Dermatol 2016;34:276-85.

32. Abdel-Aal SM, Akhtar H, Zaheer K, et al. Dietary Sources of Lutein and Zeaxanthin Carotenoids and Their Role in Eye Health. Nutrients 2013;5:1169-85.

33. Eggersdorfer M, Wyss A. Carotenoids in human nutrition and health. Arch Biochem Biophys 2018;652:18-26.

34. Stankiewicz A, Poppe E, Stasiewicz B, et al. Evaluation of the effectiveness of Quinax in the prevention of the development of senile cataract. Klin Oczna 1990;92:52-4.

35. Prost M, Toczołowski J. Clinical evaluation of the inhibitory effect of Quinax on the progression of senile cataract. Klin Oczna 1982;84:71-2.

36. Zygulska-Mach H, Pioruńska M, Paziewski E. Use of the preparation quinax in the trial treatment of senile and secondary cataract. Wiad Lek 1982;35:1239-43.

37. Bittner S. When quinones meet amino acids: Chemical, physical and biological consequences. Amino Acids 2006;30:205-24.

38. Maclean H, Webb JG, Augusteyn RC. Letter: Catalin, Quinones, and Cataract. Lancet 1974;2:895.

39. Xu GT, Zigler JS Jr, Lou MF. Establishment of a naphthalene cataract model in vitro. Exp Eye Res 1992;54:73-81.

40. Hu CC, Liao JH, Hsu KY, et al. Role of pirenoxine in the effects of catalin on in vitro ultraviolet-induced lens protein turbidity and selenite-induced cataractogenesis in vivo. Mol Vis 2011;17:1862.

41. Kociecki J, Załecki K, Wasiewicz-Rager J, et al. Evaluation of effectiveness of Catalin eyedrops in patients with presenile and senile cataract. Klin Oczna 2004;106:778-82.

42. Kinoshita JH. Mechanisms initiating cataract formation. Proctor Lecture. Invest Ophthalmol 1974;13:713-24. 
43. Kador PF, Akagi Y, Kinoshita JH. The effect of aldose reductase and its inhibition on sugar cataract formation. Metabolism 1986;35:15-9.

44. Pollreisz A, Schmidt-Erfurth U. Diabetic CataractPathogenesis, Epidemiology and Treatment. J Ophthalmol 2010;2010:608751.

45. Soni P, Choudhary R, Bodakhe SH. Effects of a novel isoflavonoid from the stem bark of Alstonia scholaris against fructose-induced experimental cataract. J Integr Med 2019;17:374-82.

46. Zhao W, Devamanoharan PS, Varma SD. Fructosemediated damage to lens alpha-crystallin: prevention by pyruvate. Biochim Biophys Acta 2000;1500:161-8.

47. Zukin LM, Pedler MG, Groman-Lupa S, et al. Aldose Reductase Inhibition Prevents Development of Posterior Capsular Opacification in an In Vivo Model of Cataract Surgery. Invest Ophthalmol Vis Sci 2018;59:3591-8.

48. Chen XJ, Hu LD, Yao K, et al. Lanosterol and 25-hydroxycholesterol dissociate crystallin aggregates isolated from cataractous human lens via different mechanisms. Biochem Biophys Res Commun 2018;506:868-73.

49. Xi YB, Chen XJ, Zhao WJ, et al. Congenital CataractCausing Mutation G129C in $\gamma$ c-Crystallin Promotes the Accumulation of Two Distinct Unfolding Intermediates That Form Highly Toxic Aggregates. J Mol Biol 2015;427:2765-81.

50. Hejtmancik JF. Ophthalmology: Cataracts dissolved. Nature 2015;523:540-1.

51. Kang H, Yang Z, Zhou R. Lanosterol Disrupts Aggregation of Human $\gamma \mathrm{D}$-Crystallin by Binding to the

Cite this article as: $\mathrm{Xu} \mathrm{J}$, Fu Q, Chen X, Yao K. Advances in pharmacotherapy of cataracts. Ann Transl Med 2020;8(22):1552. doi: $10.21037 /$ atm-20-1960
Hydrophobic Dimerization Interface. J Am Chem Soc 2018;140:8479-86.

52. Hu LD, Wang J, Chen XJ, et al. Lanosterol modulates proteostasis via dissolving cytosolic sequestosomes/ aggresome-like induced structures. Biochim Biophys Acta Mol Cell Res 2020;1867:118617.

53. Ono K, Li L, Takamura Y, et al. Phenolic compounds prevent amyloid $\beta$-protein oligomerization and synaptic dysfunction by site-specific binding. J Biol Chem 2012;287:14631-43.

54. Yamada M, Ono K, Hamaguchi T, et al. Natural Phenolic Compounds as Therapeutic and Preventive Agents for Cerebral Amyloidosis. Adv Exp Med Biol 2015;863:79-94.

55. Camilleri A, Zarb C, Caruana M, et al. Mitochondrial membrane permeabilisation by amyloid aggregates and protection by polyphenols. Biochim Biophys Acta 2013;1828:2532-43.

56. Airoldi C, Sironi E, Dias C, et al. Natural compounds against Alzheimer's disease: molecular recognition of A $\beta 1-42$ peptide by Salvia sclareoides extract and its major component, rosmarinic acid, as investigated by NMR. Chem Asian J 2013;8:596-602.

57. Tabas I. Cholesterol in health and disease. J Clin Invest 2002;110:583-90.

58. Cetinel S, Montemagno C. Nanotechnology for the prevention and treatment of cataract. Asia Pac J Ophthalmol (Phila) 2015;4:381-7.

59. Kumar B, Gupta SK, Saxena R, et al. Current trends in the pharmacotherapy of diabetic retinopathy. J Postgrad Med 2012;58:132-9. 